\title{
MEDIOS, DEBATE Y POLÍTICAS SOCIALES: PERCEPCIONES SOBRE LOS OBSTÁCULOS PARA PARTICIPAR
}

\section{MEDIA, DEBATE AND SOCIAL POLICY: PERCEPTIONS OF THE BARRIERS TO PARTICIPATE}

\author{
Elba Betancourt Díaz ${ }^{1}$ \\ elba_betancourt@yahoo.com
}

Fecha de recepción: 10 de agosto de 2015 - Fecha de aceptación: 10 de junio de 2016

\begin{abstract}
Resumen
Ser parte de la discusión pública sobre políticas sociales a través de los medios pudiera ser un desafío para los/as trabajadores y trabajadoras sociales. El reto puede deberse a múltiples factores individuales y estructurales que pudieran minar su potencial para participar en los medios e influenciar la opinión pública a favor de mejores políticas para el bienestar social. No obstante, fundamentándose en los valores de la profesión, y en sus conocimientos y experiencias, pueden ejercer diferentes roles en este debate. Sin embargo, poco se ha explorado su participación en el debate público sobre políticas a través de los medios. Esta investigación tuvo como objetivo conocer los factores que obstaculizan la participación de los profesionales del trabajo social en el debate público a través de los medios de comunicación en Puerto Rico. Se utilizó un diseño exploratorio con método mixto secuencial, en cuya fase cualitativa, se realizó entrevistas semiestructuradas a profundidad a 12 trabajadores y trabajadoras sociales. Del análisis de discurso, surgieron tres categorías vinculadas a obstáculos asociados con su proceso de socialización, dinámicas de poder al interior de sus trabajos y tensiones en la interacción con los medios. Finalmente, se ofrecen recomendaciones sobre cómo integrarse al debate público a través de los medios. Palabras claves: trabajo social, medios de comunicación, políticas públicas, debate público
\end{abstract}

\begin{abstract}
Participate in the public debate on social policies through the media could be a challenge for social work professionals. The challenge may be due to multiple individual and structural factors that could undermine its potential to access the media and influence public opinion advocating for better policies to promote social welfare. However, building on the professional values, and their knowledge and experience, social workers can exert different roles in this debate. Nevertheless, little has been explored about their participation in the public debate through the media. This research aimed to explorer the factors that hinder social workers participation in public debate on social policies through the media in Puerto Rico. To conduct the study I used a sequential exploratory mixed method design. In this article, I exposed the findings on individual and structural facilitators corresponding to the qualitative phase, in which I conducted semi-structured in-depth interviews to 12 social workers. From the analysis of discourse emerged three dimensions associated with socialization, power dynamics in theirs jobs and tension in theirs interaction with the media. Finally, I suggest recommendations on how to increase social workers participation in the public debate through the media.
\end{abstract}

Keywords: social work, media, public policy, public debate

1 Universidad de Puerto Rico (UPR). 
A través de la comunicación a nivel macrosocial se diseminan discursos públicos que alcanzan visibilidad valiéndose de los medios industriales de comunicación lo que los convierte en hegemónicos, según Von Sprecher y Boito (2010). Estos discursos subyacentes en los contenidos mediáticos moldean el pensamiento de la ciudadanía sobre cómo se definen los problemas sociales y cómo el Estado debe atenderlos. El resultado es una opinión pública que tiene la capacidad de influenciar la agenda política y viceversa. Este juego de influencias, hace necesario que profesionales del trabajo social (TS) se integren al debate público a través de los medios para infundirlo con sus conocimientos y experiencias con el objetivo ulterior de influenciar los procesos de política social (PS).

Sin embargo, acceder a los medios noticiosos pueden ser un desafío para los/as profesionales del TS. El reto puede deberse a múltiples factores individuales y estructurales que pueden minar su potencial para sumar sus perspectivas a las discusiones que influencian la opinión pública. No obstante, de acuerdo con Guardiola y Sierra Taylor (2002), fundamentándose en los valores de justicia y equidad social de la profesión, se ha reconocido que pueden desempeñarse como comunicadores/as, intercesores/ as, persuasores/as y colaboradores/as en los procesos de PS. Como plantean Weiss-Gal y Peled (2007), los/as trabajadores y trabajadoras sociales tienen como fortaleza para incursionar con éxito a nivel macrosocial la perspectiva cercana que su trabajo les brinda de los problemas sociales, y de los efectos positivos y negativos de las PS. Muestra de ello son los resultados de un estudio sobre su participación en tres comités del parlamento israelí, el cual concluye que estos profesionales facilitan y enriquecen el proceso de formulación de las políticas públicas ofreciendo sus conocimientos y experiencias sobre temas sociales. Además, como establecen Weiss-Gal y Gal (2014), aquellos/as que trabajan en organizaciones sin fines de lucro, retan a los/as hacedores de política pública introduciendo temas en las agendas de los comités. Estas incursiones y prácticas demuestran la capacidad de ejercer como actores políticos en la formulación de política pública de estos profesionales.

Otra forma de integrarse al nivel macrosocial es concebir los medios como un escenario más de acción política. Como plantean Von Sprecher y Boito (2010), la comunicación debe servir para aprovechar los reducidos espacios de libertad, un poder fundamental en las luchas sociales y una manera de generar espacios contra la dominación y el sentido común imperante dentro de una hegemonía.

Este planteamiento no anula el hecho de que, como plantea Vallespín (2000), el acceso a los medios, que en su mayoría pertenecen al sector privado, sea asimétrico para los/as miembros de una sociedad. Usualmente, los medios dan espacios y visibilidad a ciertos sectores o líderes/lideresas de opinión; mientras silencia o invisibiliza a otros/as. Esta dinámica de los medios tiene efectos adversos como banalizar los discursos públicos y distorsionar la opinión pública. No obstante, para Andrade del Cid (2009) y Vallespín (2000) los medios noticiosos siguen siendo ese espacio público donde se debate sobre política, ventilan ideas, construyen sentidos sobre los asuntos públicos y se difunden opiniones.

Los estudios realizados en Puerto Rico por Canino-Arroyo y colegas (2008) y Guardiola y Sierra Taylor (2002) sobre la participación de los/as profesionales del TS en la PS dan cuenta de la pertinencia de que estos/as se inmiscuya más en estos procesos y la existencia de un desfase entre el deber de participar y concretar la acción. Por otro lado, al explorar cómo es la relación de estos/as profesionales con los medios, la literatura evidencia que existe una tensión porque los/as trabajadores/as sociales consideran se presenta una imagen negativa de ellos/as; y, por su parte, los/as periodistas, creen que a los/ as trabajadores/as sociales les faltan de destrezas para manejarse ante los medios (Ayre, 2001; Freeman \& Valentine, 2004; Gaughan \& Garrett, 2012; Mendes, 2001; Zurazaga, Surette, Méndez \& Otto, 2006). Otra investigación, apunta a su fracaso para influenciar la prensa y al público en el nivel de las ideas (Aldridge, 1990). Sin embargo, para lidiar con esta situación se han realizado esfuerzos de algunas universidades para desarrollar en el estudiantado de TS destrezas para manejar los medios (Stone, Ekman, English \& Fujimori, 2008; Weiss-Gal \& Peled, 2009). Igualmente, otros estudios señalan la necesidad de que desarrollen destrezas para debatir, argumentación, persuasión e 'intercesoría' mediática para influenciar la agenda pública y a los/as hacedores/as de política pública (Brawley, 1997; Curry-Stevens, 
2012; Gregory \& Holloway, 2005; Lens, 2005). No obstante, existe un vacío en cuanto a investigaciones que examinen los factores que fomentan y obstaculizan su participación en el debate público sobre PS.

Esta investigación se fundamentó en el paradigma socioconstruccionista. De acuerdo con Berger y Luckmann (1966) la realidad es una construcción colectiva. Bajo la mirada del socioconstruccionismo se reconoce que la realidad es múltiple; que se transforma mediante la construcción y reconstrucción de esos discursos; y se toman en cuenta múltiples voces que interactúan en la concepción de lo que se entiende como realidad. De acuerdo con Gergen (1999), el significado de nuestro mundo es generado a través de la forma en que colectivamente usamos las palabras. Los medios son una de las vías por las cuales se normalizan discursos, construyen imaginarios y se naturaliza la realidad social. Estos influyen la producción y distribución de conocimiento que da sentido a la experiencia del mundo social, legitima la historia, establece el presente, define la identidad cultural y presenta estándares, modelos y normas para garantizar una vida pública y social en común.

Determinar con precisión cuáles y cómo son los efectos de los medios en los públicos siempre ha sido un reto. Entre los efectos que se han documentado están los producidos por el agenda setting o establecimiento de una agenda temática o jerarquización de noticias. Este proceso ha sido descrito por McCombs (1997) y Rubio Ferreres (2009) como el establecimiento (deliberado o no) de la relevancia de un grupo de temas o eventos; el grado de prioridad o exposición que se les otorga en el medio; la repetición con la que se publican noticias sobre estos temas o eventos, y el contexto (framed) en el que se presentan. Como plantea Andrade del Cid (2009), la pretensión de los medios con su contenido informativo es "mover a la opinión pública al determinar, por emisión o por omisión, los temas que se debaten. Es un juego de poder institucional, político y cultural, que consiste en definir y jerarquizar los temas que discute la opinión" (p. 92).

Sobre la simbiosis entre este proceso y el campo político, Chomsky (s.f.) plantea que los medios sirven para transmitir mensajes propagandísticos elaborados por una élite para fabricar la opinión pública y el consenso sobre ciertos asuntos a través de falsear la realidad y crear potenciales enemigos de la nación, mientras los/as espectadores/as (la mayoría de la ciudadanía) permanecen inmóviles ante los medios sin permitírseles participar de procesos realmente democráticos.

Rogers y Dearing (1994) en su modelo sobre el proceso de establecimiento de una agenda de noticias diferencian entre la agenda de los medios, la agenda pública y la agenda política. La primera se refiere a la relevancia, exposición, prioridad y repetición de ciertas noticias en los medios. La segunda se relaciona con el contenido y orden de los temas en la agenda pública (es decir, la opinión pública). Mientras que la agenda política se enfoca en la influencia de la agenda de los medios y la del público en los procesos de política pública. Estos tres procesos se interrelacionan e influencian los medios, la opinión pública y el desarrollo de las políticas públicas.

Como plantean Jiménez, Mayers, Chambers y Pearlman (2015) es en esta interrelación de influencias sobre el desarrollo de PS, los/as trabajadores y trabajadoras sociales deben intervenir pensando en el bienestar social y de las poblaciones con las que trabajan pues son estas políticas las que informan, limitan o adelantan la práctica del TS y el objetivo de la justicia social. Si, como menciona Rozas (s.f.), se considera que las PS, son una definición estratégica de todo desarrollo, y un estructurador de la ciudadanía y de los derechos sociales, su intervención en cada etapa del proceso de política pública es inminentemente necesaria. Como menciona Guardiola (2002), el apoderamiento del proceso de formulación de política pública es una vuelta a las raíces políticas de la profesión. Ser parte de este proceso es fundamental, sobre todo en el contexto de las políticas de corte neoliberal, las cuales promueven un grado de mercantilización de los bienes sociales y someten su prestación a una lógica de lucro.

Esta participación se apoya en la justicia social y dignidad humana, valores profesionales que están contenidos en varios códigos de ética profesionales (CPTSPR, 2011; FITS \& AITS, 2004; NASW, 2008). Estos valores les comprometen a promover y luchar por la transformación social que redunde en beneficio de las poblaciones oprimidas y vulnerables, fomentando su fortalecimiento e inclusión social. El Código de Ética de la Federación Internacional de Trabajo Social y de la Asociación de Escuelas de Trabajo Social (2004) establece acciones concretas relacionadas con el proceso de política pública, 
como: llamar la atención de empleadores/as, la legislatura y la clase política y de la sociedad sobre situaciones en las que los recursos son inadecuados o cuando la distribución de recursos, políticas y prácticas son opresivas, injustas o perjudiciales. Así como también exhorta a oponerse a situaciones que contribuyen a la exclusión social, estigmatización o subyugación y trabajar por una sociedad inclusiva.

Estas acciones podrían constituir parte de su intervención en el debate público para influenciar la agenda de los medios, la opinión pública y a los/as hacedores/as de política pública en pro de las poblaciones excluidas que no tienen las posibilidades de ser emisores/as en los medios. Así, los/as profesionales del TS serían parte de la dinámica de influjos que informan la formulación, aprobación e implementación de las PS (ver Diagrama 1).

Diagrama 1

Interrelación de influencias sobre la opinión pública y la PS

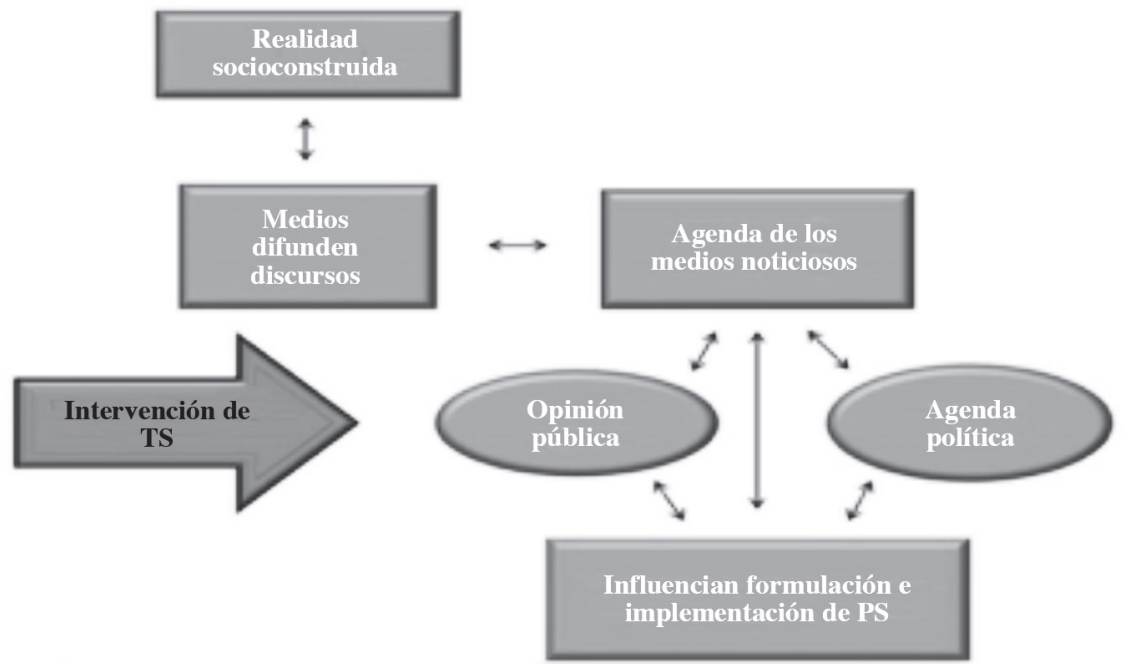

Tabla: Elaboración propia.

Para conocer más sobre la relación de estos/as profesionales y el debate a través de los medios, este estudio tuvo como objetivo conocer los factores individuales (experiencias de vida y formativas, socialización, adiestramientos) y estructurales (percepción del Colegio de Profesionales del Trabajo Social de Puerto Rico (CPTSPR), de los medios) que obstaculizan su participación.

\section{MÉTODO}

Para realizar el estudio se utilizó un diseño de métodos mixtos (Creswell, 2003). En este artículo se presenta el método de la parte cualitativa de la cual emanaron los resultados que expongo. En esta etapa, se realizaron entrevistas a profundidad semiestructuradas a 12 trabajadores y trabajadoras sociales, seis (6) que habían participado en el debate público sobre PS a través de los medios y seis (6) que no habían participado.

\section{Participantes}

Los/as 12 participantes fueron reclutados/as por disponibilidad y eran mayores de edad: seis (6) habían participado del debate público a través de los medios y seis (6) no. Los/as seis (6) trabajadores/ 
as sociales que habían participado fueron identificados/as durante el análisis de contenido que se realizó en la fase cuantitativa de este estudio. A los seis (6) que más aparecieron entrevistados/as o como autores/as de artículos de opinión se les envió una carta de invitación para participar del estudio. Todos/ as aceptaron ser entrevistados/as. Estos/as profesionales provenían de la academia y/o habían ocupado puestos de liderato en el CPTSPR o en una dependencia gubernamental.

Los/as restantes seis (6) que no habían participado en el debate público fueron reclutados/as a en las escuelas graduadas de TS de universidades, en el CPTSPR y en el Departamento de la Familia (DF) (agencia encargada de los servicios sociales) (Ver Tabla 1).

Tabla 1

Descripción de los/as participantes

\begin{tabular}{cccc}
\hline Trabajador/a Social & Grado & Años de Experiencia & Puesto que ocupa \\
\hline TS1P & Doctorado Sociología & 37 & Retirado \\
\hline TS2P & Doctorado TS & 25 & Catedrática \\
\hline TS3P & Doc. Sicología (en progreso) & 15 & Catedrático \\
\hline TS4P & Doctorado TS & Más de 30 & Práctica Privada \\
\hline TS5P & Juris Doctor & 37 & Catedrática \\
\hline TS6P & Maestría TS y Adm. Escolar & 28 & TS Escolar \\
\hline TS7NP 2 & Maestría TS & 12 & TS Salud Mental \\
\hline TS8NP & Maestría Orientación & 22 & TS Depto. Familia \\
\hline TS9NP & Bachillerato TS & 15 & TS Depto. Familia \\
\hline TS10NP & Maestría TS (en progreso) & 5 & TS Depto. Familia \\
\hline TS11NP & Maestría TS & 3 & Manejadora casos \\
\hline TS12NP & Estudiante doc. Psicología & 5 & Interna en Psicología \\
\hline
\end{tabular}

Tabla: Elaboración propia.

\section{Procedimiento}

El protocolo de investigación fue autorizado por el Comité Institucional para la Protección de los Seres Humanos en la investigación de la Universidad de Puerto Rico. Tras la aprobación, se comenzó a invitar a potenciales participantes. Todos/as los/as entrevistados/as firmaron la hoja de consentimiento que se discutió con ellos/as antes de la entrevista. A las personas que no habían participado del debate, les ofreció confidencialidad; mientras, que a las personas identificables por sus temas de trabajo o exposición en los medios, les expliqué que no les aseguraba esta confidencialidad. Las entrevistas fueron grabadas y duraron entre 20 minutos a una hora. Para asegurar la confidencialidad se utilizaron códigos numéricos en la hoja de consentimiento, grabaciones y transcripciones.

2 TS1P: Se refiere a trabajadores/as sociales que participaron del debate y el orden de la entrevista.

3 TS2NP: Se refiere a trabajadores/as sociales que no participaron del debate y el orden de la entrevista. 


\section{Instrumento}

Para recopilar los datos se usaron dos guías de preguntas, una dirigida a las personas que habían participado del debate y otras a las que no habían participado. Las preguntas abiertas giraron en torno a: experiencias de participación en el debate; factores individuales que fomentan la participación; factores estructurales que obstaculizan la participación y estrategias para potenciar la participación.

\section{Análisis}

Se transcribieron las entrevistas a verbatium utilizando el programado HyperTranscribe para el posterior análisis de discurso. El proceso de análisis se inició haciendo anotaciones sobre ideas, patrones y temas que surgieron en las entrevistas (Potter, 2009). Posteriormente, desarrollé dimensiones temáticas, categorías y subcategorías que me facilitarán trabajar con una gran cantidad de datos textuales, como establecen Potter (2009) y Potter y Wetherell (1987). Las dimensiones, categorías y subcategorías están esbozadas en la Tabla 2.

Tabla 2

Descripción de las dimensiones, categorías y subcategoría

\section{Socialización de los/as trabajadores/as sociales}

Asuntos relacionados con cómo son socializados/ as durante su formación académica y su desarrollo profesional.

Dinámicas de poder al interior de sus trabajos

Asuntos sobre el juego de poder que subyace a la forma en que se organizan las agencias/organizaciones donde trabajan y las implicaciones en su participación.
Falta de motivación/Interés- Comentarios sobre factores relacionados con interés, compromiso y atreverse participar

Pobre percepción/imagen de su profesión- Verbalizaciones sobre cómo la pobre imagen y percepción que algunos/as tienen de la profesión subestima su habilidad para participar.

Pobre percepción /imagen que otros/as tienen del TSExpresiones sobre cómo la pobre percepción e imagen que otros/ as tienen de la profesión incide en si los invitan a participar.

Condiciones de trabajo- Expresiones sobre cómo el puesto que ocupen, dónde trabajen y las condiciones de trabajo impiden que participen del debate.

Políticas internas de las agencias u organizacionesComentarios sobre cómo las agencias u organizaciones establecen normas sobre quiénes pueden expresarse en los medios.

Repercusiones a nivel personal y/o profesional- Expresiones sobre acciones/situaciones adversas a nivel personal y/o profesional que pueden ocurrir como consecuencia de su participación. 


\section{Tensión en la interacción con los medios}

Asuntos sobre cómo describen y perciben su relación con los medios y cómo esto repercute en su participación.
Falta de destrezas - Verbalizaciones relacionadas con no poseer conocimientos sobre cómo funcionan los medios, cómo conseguir espacios para publicar o saber cómo expresarse a través de estos.

Falta de disponibilidad- Expresiones sobre no disponer de tiempo por carga de trabajo y otras responsabilidades personales.

Falta de espacios en los medios- Expresiones sobre dificultades para acceder a espacios en los medios para participar del debate.

Trabajo periodístico- Expresiones sobre factores asociados a la naturaleza del trabajo en los medios noticiosos que dificultan la participación.

\section{Subcategorías}

Errores de publicación- Asuntos relacionados con posibles errores que pueden cometer los/as periodistas.

Elección de las fuentes de información- Asuntos relacionados con las personas que selecciona el/la periodista para entrevistar.

Tratamiento de los temas- Asuntos sobre los ángulos que los/ as periodistas o los medios dan a los escritos.

Factor sorpresa- Asuntos sobre que no expliquen con anterioridad los temas a ser tratado en la entrevista.

Restricciones de los medios- Asuntos sobre las limitaciones o reglas que el medio impone.

Tabla: Elaboración propia.

\section{Resultados}

A continuación, por razones de espacio, se detallan solo algunas categorías y verbalizaciones.

Falta de destrezas. Algunos retos que han enfrentado cuando desean intervenir en los medios es desconocer cómo estos funcionan, no saber cómo conseguir espacios o no poseer destrezas para expresarse adecuadamente. Un entrevistado señaló la dificultad que enfrentan algunos/as colegas: "Pero es que hay personas que no le gusta hacer eso [presentarse en los medios] porque argumentan que se sienten nerviosas. Que se sienten cuestionadas" (TS1-P). Sobre su experiencia comenta que: "Fue una buena experiencia. El único problema que tuve fue la limitación de palabras” (TS1-P).

Otras participantes no recordaron que les enseñaran destrezas para trabajar con los medios. Una de ellas, resaltó en esta verbalización la necesidad de incorporar estos temas para sentirse más seguros/ as al participar en el debate sobre políticas sociales. 
Nos hacen falta más herramientas prácticas de cómo abordar el escenario político. Yo creo que como que los trabajadores sociales somos tímidos y sentimos que necesitamos más herramientas. [...] Tenemos ideológicamente la noción, pero pienso que a veces nos sentimos inseguros porque nos falta más conocimiento y [eso] nos intimida (TS12-NP).

Falta de disponibilidad. No contar con tiempo para redactar o presentarse en un medio fue un obstáculo, sobre todo para quienes trabajan en servicios directos. Esta percepción la comparten tanto quienes participan en el debate, como quienes no lo han hecho. Las razones incluyen: responsabilidades personales y laborales, la inmediatez con la que trabajan los periodistas, las condiciones de trabajo y tener otros empleos. Así lo expresó esta participante: "Bueno, el tiempo. Tenemos varios roles profesionales, padres, familia, hijos y, eso pues, la falta de tiempo nos roba un poco la posibilidad de escribir" (TS3-P).

Por su parte, al preguntarle a esta participante, empleada de una agencia gubernamental, por qué no se involucra en el debate también se refirió al tiempo y cómo sus condiciones de trabajo se convierten en obstáculos para realizar otras actividades: "Pues más bien es por factor tiempo. No tener el tiempo, son horario rotativos, siete días a la semana, 365 días al año. La oficina no cierra, no hay una hora de salida establecida" (TS9-NP).

Pobre percepción/imagen de su profesión. Algunos/as entrevistados/as expresaron cómo la pobre imagen que se ha desarrollado de la profesión, y que hasta cierto punto ha sido asumida por algunos sectores de los/as profesionales, les cohíbe de participar en el debate público y les hace dudar sobre sus habilidades para influenciar el debate. A ello, se suma la falta de consenso en el gremio sobre cómo se define el TS y el alcance de sus intervenciones, asunto que contribuye a ver la profesión como una supeditada a otras disciplinas y a la distorsión del rol que el/la profesional desarrolla de su intervención en los procesos de PS. "Pero hay gente que dice que somos una, cómo es que dicen una disciplina técnica. Disciplina técnica es lo que hace una de mis nietas...la gimnasia [...] Eh, pues mientras nosotros estemos con esas monerías, pues vamos a estar que no sabemos ni lo que somos" (TS4-P).

En este renglón, se mencionaron cuestiones en torno a la opresión institucional como factor que afecta adversamente su estima como profesionales y les hace sentirse menos seguros/as al hacer expresiones públicas y participar del debate. La siguiente verbalización es una ejemplo de esto: "Mira, por ejemplo el sueldo que tú devengas aquí...Mira, yo he estado con dueños de puntos de drogas. Me han sacado armas de fuego. [...] Y cuando llegas a la oficina lo que encuentras son personas, que posiblemente, te tratan mal. A veces no sientes un respaldo y apoyo... lo que tú necesitas. Más cuando llega tu cheque [haciendo referencia al salario bajo]... tú te das cuenta que estás nadando en contra de la corriente. Entonces, ¿qué motivación o qué estima puede tener un profesional en una situación como esta? Con qué standing, como dicen los americanos, una persona se va parar y decir a mucha honra y orgullo: 'Soy un trabajador social"' (TS10-NP).

A continuación esbozo algunas de las categorías que emergieron relacionadas con factores estructurales.

Pobre percepción /imagen que otros/as tienen del TS. La percepción o imagen que la sociedad y profesionales de los medios tienen del TS, según sus experiencias, afecta negativamente la posibilidad de que sean considerados/as como fuentes en sus noticias o como colaboradores/as. Las verbalizaciones reflejan que existe en el imaginario social una percepción o imagen negativa por las tareas que realizan en ciertas agencias gubernamentales, reforzando la noción de que no están capacitados/as o no tienen las habilidades para aportar en otros ámbitos.

Este participante menciona experiencias con medios que prefieren identificarles con sus otros títulos académicos, pues consideran que tienen más estima o credibilidad. No obstante, él insiste en que lo identifiquen como trabajador social para darle visibilidad a la profesión: "Ellos entienden que es más importante decir que tú eres criminólogo. Sociólogo, también les llama la atención. Y psicólogos también les gusta mucho. Pero trabajador social, no. Psicólogo les encanta" (TS1-P). 
Otro participante describe con la siguiente verbalización cómo los/as profesionales del campo lidian con esa percepción negativa cuando tienen una participación pública en los medios.

\begin{abstract}
Mucha de esa gente [trabajadores/as sociales] está luchando con el discurso que hay en la calle: 'Tu profesión no es lo suficiente'. Entonces, ya cuando llegan allí [a expresarse públicamente], lo que tienen que hacer es, darle un makeover a su corazón, a su mente, para sentirse como que pueden sacar un poquito el pecho. Pero, realmente, tú sabes muy bien que tú llegas a los lugares y tú dices: 'Soy maestro', 'Soy Trabajador Social', y la gente se ríe: 'Qué bueno, qué bueno'. Pero tú sabes que cuando va a dar media vuelta va a decir: 'Ay, bendito, tan inteligente' (TS9-NP).
\end{abstract}

Fuentes de información. Para algunos/as participantes los/as periodistas suelen entrevistar a las mismas fuentes, lo que reduce su posibilidad de participar. Resulta interesante la aseveración que hace uno de los participantes sobre que entrevisten a personas porque llaman la atención por sus estilos, pero no necesariamente porque tengan la combinación de conocimientos y experiencia que tiene un/a trabajador/a social.

Entonces tenemos una persona, por ejemplo un Tito $\mathrm{Kayak}^{4}$, una persona que apenas creo sabe expresarse, pero tiene más público. Obviamente, yo admiro igualmente su capacidad de poder llamar la atención de las masas. [...] Más criticaría a un Papo Christian ${ }^{5}$, que sí que habla súper malo. Y entonces parece que uno tuviera que vestirse de payaso para que la gente te escuche (TS10-NP).

Esta participante, que labora en el DF, opina que cuando los/as periodistas entrevistan buscan a las fuentes que ya conocen o los/as supervisores/as de áreas porque es más rápido. Sin embargo, considera que no se esfuerzan por conseguir otras fuentes, como pueden ser los/as investigadores/as. Así lo expresó durante la entrevista:

Este, a lo mejor necesitan rápidamente... este, buscar a alguien, pues más rápido, el que yo conozco. [...] Pero, como dice usted, el venir aquí... el verificar algún tipo de investigador. Porque toda esta parte de arriba, estamos hablando de un montón de directores regionales que usted puede entrevistar, pero son todas ellas de maestría y doctorado, que están como supervisoras regionales. Pero los que estamos abajo que somos Trabajadores Sociales de field, ya eso es otra área (TS8-NP).

Restricciones de los medios. De acuerdo con algunos/as entrevistados/as, los medios como parte de la inmediatez y dinámica de trabajo imponen exigencias que dificultan su participación y crean tensiones en su relación. Una de las restricciones que comentaron fue el espacio para escribir los artículos de opinión o para expresarse en los medios audiovisuales. La siguiente verbalización resume la experiencia de un participante:

Cuando te dicen: 'Tienes 700 palabras para responder a esto'. Dices: 'Anda, lo voy a intentar'. Yo en las ocasiones comencé a escribir y cuando le doy para que me cuente las palabras: 905, 800 tantas. ¡Ay, Dios mío! Pero, es que esto no lo puedo decir de esta forma ni de esta. Yo no sé cómo decirlo. Entonces desistí porque sentía que esa presión no me permitía ser más libre en mi expresión (TS1-P).

Los/as entrevistados/as también mencionaron que a veces no les publican sus aportaciones, lo que resulta frustrante y lacera las relaciones con los/as editores/as, pues parecería que toman las decisiones de forma arbitraria. A continuación la vivencia de una de las entrevistadas:

4 Líder ambientalista reconocido por sus manifestaciones inusuales.

5 Líder comunitario que los medios suelen entrevistar. Es reconocido por su estilo de expresarse sin tapujos. 
Y tú lo llamabas o te decían: 'Sí, sí, sí está excelente. Se va a publicar'. Y no pasaba, y no pasaba. Esa cuestión [de no publicar] un poco caprichosa del editor, sin que tuviera unos criterios claros, después que tú tuviste cinco o seis horas [escribiendo]. Tiempo que no te sobra, pues puede provocar un poco de molestia (TS5-P).

Repercusiones a nivel personal y profesional. Otra dificultad que mencionaron los/as participantes fueron las repercusiones a nivel profesional o personal a las que pudieran enfrentarse. A estas consecuencias les subyacen dinámicas de poder en sus empleos, prejuicios, estigmas o la influencia de la política partidista. Esta entrevistada narró en esta verbalización una experiencia que le ocurrió con una colega para ejemplificar las repercusiones a nivel personal de sus expresiones sobre el tema de las adicciones a drogas ilegales.

Su expresión no verbal; todo era el desprecio de: 'Mija, tantas cosas que tú tienes que hacer, mira con lo que tú te has quedado'. Y entonces más valor le das. A mí no me importa, pero me han estigmatizado. Nos dicen también: 'Mira, la gente esa que andan defendiendo a los tecatos ${ }^{6}$. A esos, presos los deberían meter también' (TS2-P).

Este otro participante, que suele publicar columnas de opinión, contó cómo le afectó una crítica que hizo a un partido político.

Tuve una consecuencia por un artículo: daba servicio voluntario en un centro de personas con adicción. Ese centro era manejado por un municipio y yo critiqué un [a] político del partido de gobierno de ese municipio y de ahí en adelante comenzó una falta de contacto con este servidor para el proceso de ayuda que yo daba allí. Hasta que me dijeron que no hacía falta allí, que no tenía que ir más (TS3-P).

\section{Discusión}

En esta sección discutiré las tres dimensiones analíticas amplias para arrojar luz sobre cómo sobrepasar estos obstáculos.

\section{Socialización de los/as trabajadores/as sociales}

La socialización de los/as profesionales del TS durante su formación y desarrollo profesional debe reforzar el sentido político de la profesión y la pertinencia de su intervención en el nivel macrosocial para transformar las políticas y estructuras sociales opresivas. Como plantean Velasco Vázquez (2012) y Weiss-Gal y Gal (2009), Los currículos universitarios deben alejarse de la visión asistencialista de la prestación de servicios para desarrollar en el estudiantado una conciencia crítica que promueva y motive su participación en el escenario político y enfatizar en el rol que pueden desempeñar como intercesores/as junto con las poblaciones con quienes trabajan. Según Seda (2009), cuando se tiene conciencia crítica, la intervención profesional se enfoca en interpretar los problemas sociales desde las macroestructuras que los generan y perpetúan y no desde los microniveles personas y familiares. Este tipo de análisis es el que deben aportar para guiar la discusión hacia la dimensión de las PS que son las que inciden en las macroestructuras que deben ser transformadas.

No obstante, Fook (2002) plantea que los/as trabajadores y trabajadoras sociales piensan que carecen de poder y de una voz, y que no son capaces de afectar la realidad social, noción que debe ser transformada a través de socialización; de igual modo, este planteamiento fue mencionado durante las entrevistas. Los/as profesionales en TS deben empoderarse para cambiar la apreciación que hacen de sí mismos/as en relación con los medios. Como surgió en algunas de las entrevistas, es necesario trabajar con el hecho de que hay trabajadores y trabajadoras sociales que pueden concebir la profesión como 
una subordinada a otras disciplinas o a las estructuras estatales, lo que limita su interés y potencial de participar en el debate público a través de los medios.

Los currículos de las escuelas de TS deben incluir en todos los niveles cursos que vinculen los procesos de PS y los medios. Así como, cursos o talleres sobre cómo debatir y argumentar, y sobre 'intercesoría' mediática. Con estas destrezas podrán posicionar temas dentro de la agenda pública para educar a la sociedad, a los líderes y las lideresas de opinión y a los/as hacedores/as de política pública. Como establece Brawley (1997), deben seguir los preceptos de la 'intercesoría' mediática, la cual se fundamenta más en explicar los factores estructurales que inciden en los problemas sociales que en los individuales, lo que cambia la perspectiva de la discusión pública. Estas clases que incorporan el aspecto macrosocial de la profesión, como sugieren Mink y Twill (2012), deben basarse en un modelo de aprendizaje-servicio que incorpore la práctica de las destrezas que pretenden enseñar para aumentar las actitudes positivas, la participación, la confianza y la competencia en el desempeño a nivel macro.

Un punto neurálgico en esta discusión es realzar la imagen y autoconcepto del/a trabajador/a social. Como plantean Zurazaga y colegas (2006), la representación negativa que se hace de ellos/as influye adversamente en lo que piensan de sí mismos/as, su moral y desempeño laboral; así como en la opinión pública sobre el trabajo que realizan y sus capacidades. Esta imagen construida de la profesión y que ha sido diseminada a través de los medios también ha influenciado a la sociedad. Por esto, es necesario que diseñen estrategias para mostrar otra cara del TS que ha sido invisibilizada. Una forma de hacerlo es expresándose a través de los medios para que la profesión gane reconocimiento. Otro esfuerzo puede ser desarrollar campañas de relaciones públicas que muestren sus contribuciones, así como el compromiso que tienen con la transformación social.

\section{Dinámicas de poder al interior de sus trabajos}

Las organizaciones donde trabajan, sus actitudes y políticas han demostrado tener influencias en cómo se desempeñan estos profesionales. Weiss-Gal y Gal (2009), en un estudio realizado en Israel sobre 'intercesoría' y profesionales en TS concluyeron que la práctica de la profesión está más influenciada por las políticas de las organizaciones que quienes los/as emplean que las actitudes individuales y conocimientos percibidos de esto/as profesionales. Para aumentar la práctica de la 'intercesoría', los investigadores enfatizan la necesidad de cambiar la percepción de los/as trabajadores y trabajadoras sociales y de los/as administradores/as de las organizaciones de bienestar social. Como vemos las dinámicas laborales no deben ser obviadas cuando se trata de comprender la práctica del TS en cualquiera de sus niveles de intervención.

Ahora bien, del análisis de las entrevistas emanó que existen al interior de sus trabajos dinámicas de poder que pueden ser obstáculos para su participación, como: opresión institucional, políticas organizacionales restrictivas y aprensión sobre posibles repercusiones adversas a nivel personal y profesional. Deben discutirse los asuntos relacionados con las políticas y actitudes de las agencias públicas u organizaciones privadas sobre expresarse públicamente, puesto que no debería limitarse su participación en el debate sobre PS relacionadas con su área de peritaje. Igualmente, se debe permitir participar en entrevistas a trabajadores y trabajadoras sociales que no ocupan puestos directivos, quienes lidian a diario con la realidad social y las implicaciones de las PS. La diferenciación jerárquica fue vista por algunos/ as participantes como una exclusión elitista, y una subestimación de sus conocimientos y aportaciones.

Esta reflexión también debe incluir asuntos relacionados con cómo lidiar con las repercusiones a nivel profesional y personal que puede producir su participación, sobre todo cuando son empleados/ as por el Estado. La aprensión por las posibles consecuencias negativas puede redundar en autocensura por temor a perder sus trabajos, credibilidad o respeto ante sus colegas. Esta situación puede crearles una disputa interna sobre su responsabilidad profesional con sus participantes o la transformación social versus su seguridad laboral o ser aceptado en ciertos círculos gremiales. 
Asimismo, es necesario atender las condiciones en las que se desempeñan, las cuales limitan su tiempo, pues algunos/as requieren tener más de un empleo para subsistir. Zhao y Fan (2013) plantean que para desarrollar talentos en el área de TS y motivarlos/as se requiere poner en práctica funciones de recursos humanos multidimensionales que integren de forma dinámica: la selección del personal, la evaluación de su desempeño, el salario que reciben y el adiestramiento que se les ofrece. Además, establecen que la construcción que culturalmente se hace de la profesión es un incentivo moral para que reconozcan el valor de su contribución a la sociedad, y también les ayuda a alcanzar sus metas.

Esta dimensión produce interrogantes que requieren ser caviladas y contestadas al interior del gremio. ¿Cómo los/as profesionales del TS pueden armonizar sus responsabilidades y exigencias laborales, con el deber y su deseo de participar en el nivel macrosocial, como por ejemplo en los procesos de PS? ¿Cómo se integra al debate público a través de los medios; como parte de su desempeño laboral o en su carácter individual? ¿Dónde se traza la frontera entre un escenario y el otro? ¿Qué repercusiones tiene participar desde cada uno de estos?

\section{Tensión en la interacción con los medios}

La relación entre los medios y profesionales del TS bien podría describirse como el enfrentamiento entre un gigante, controlador del acceso a la plataforma mediática y su contenido, y un enano que se esfuerza por alzar su voz para que sea incluida. La dinámica de poder, sin duda, es desigual y son los medios comerciales quienes sientan las pautas. A esta asimetría de poderes, se suma el arraigamiento del sensacionalismo en la cobertura periodística y la tendencia a publicar seudonoticias para captar la atención de más lectores/as. Este tipo de cobertura responde más a los intereses económicos de los/as dueños/as de los medios que a la función social del periodismo. Bajo este esquema mercantilista ha quedado relegada la responsabilidad periodística de informar a los/as lectores para que piensen críticamente y tomen decisiones acertadas en cuanto a los asuntos sociales, económicos y políticos.

Este contexto árido puede desalentar la participación; sin embargo, como se evidenció en este estudio no es imposible si desarrollan las destrezas, y si se tiene interés y compromiso. Durante su formación deben conocer la dinámica de los medios noticiosos impresos, digitales y audiovisuales, saber hacer una lectura crítica de las informaciones difundidas, aprender a redactar o expresarse en los medios, conocer estrategias de relaciones públicas, educarse sobre las nuevas tecnologías, aprender a desarrollar medios alternativos y saber cómo utilizar las redes sociales como medio para promover ideas, movimientos o para realzar su imagen ${ }^{7}$. También, se deben fomentar intercambios y colaboraciones con las escuelas de Comunicación y los medios para promover el entendimiento de la naturaleza de ambas disciplinas y el respeto profesional. Un ejemplo de este tipo de relación lo esboza Montoya Lodoño (2009) en su trabajo sobre los medios y las organizaciones de la sociedad civil y su alianza en pro de la democracia y los derechos humanos en Colombia.

Y una vez logren hacerle una fisura a esa relación desigual que permita acceder a los medios, deben elevar las discusiones al nivel de las PS desde la perspectiva del TS y hacer un análisis crítico de los fenómenos sociales. La interacción con los medios debe ser para integrar puntos de vista alternativos a los discursos homogenizadores, oficiales y hegemónicos, de modo que se enriquezca el análisis y se pongan de manifiesto contradicciones, complejidades y fallas que otros/as prefieren no discutir. Esto pueden hacerlo durante las entrevistas que le hagan los medios y redactando artículos en las secciones de opinión, espacios creados para que personas de diferentes trasfondos personales, profesionales y/o ideológicos se expresen más libremente sobre un tema (esto no quiere decir que el medio no filtre el contenido). Igualmente, pueden desarrollar sus propios medios valiéndose de las nuevas plataformas tecnológicas y las redes sociales para liberarse de las restricciones o filtros que imponen los medios tradicionales.

7 Para más recomendaciones sugiero leer el artículo: Betancourt-Díaz, E. (2012). Influencia de los medios noticiosos en el desarrollo de PS: Implicaciones para el Trabajo Social. Revista Análisis, 13. 


\section{Bibliografía}

Andrade del Cid, Patricia. (2009). La democracia en el espacio público mediático. Global Media Journal Edición Iberoamericana, 6 (12) 91-100.

Aldridge, Meryl. (1990). Social Work and News Media: A Hopeless Case? The British Journal of Social Work, 20(6), 611-625.

Ayre, Patrick. (2001). Child Protection and the Media: A Lesson for the Three Decades. British Journal of Social Work, 31, 887-901.

Berger, Pete y Luckmann, Thomas. (1966). The Social Construction of Reality. USA: Anchor Books.

Brawley, Edward. Allan. (1997). Teaching social work students use advocacy skills through the mass media. Journal of Social Work Education, 33, 445-60.

Canino- Arroyo, María Josefa; Lugo-Hernández, Eduardo; Díaz-Meléndez, Lymari; Vélez- Pérez, D., Carvallo-Messa, Verónica y Serrano-García, Irma. (2008). Encuesta de la participación de trabajadores y trabajadoras sociales en la política pública en Puerto Rico. En Nilsa Burgos Ortiz y Jorge Benítez Nazario (Eds.), Política Social y TS: Comunidades y PS entre la académica y la práctica cotidiana. Serie Atlantea, 4, 67-87.

Chomsky, Noam. (s.f.). El control de los medios de comunicación. Recuperado de https://docs.google. com/file/d/.../edit?usp=sharing

CPTSPR. (2011). Código de Ética de TS. San Juan: Autor.

Creswell, John. (2003). Research Design Qualitative, Quantitative and Mixed Methods Approaches. Thousand Oaks, CA: Sage.

Curry-Stevens, Ann. (2012). Persuasion: Infusing Advocacy Practice with Insights from Anti-Oppression Practice, Journal of Social Work, 12(4), 345-363.

FITS y AETS. (2004). Código de Ética. Recuperado de http://www3.unileon.es/ce/ets/ficheros/Codigo_ FITS.pdf

Fook, Jan (2002). Social Work: Critical Theory and Practice. Londres: Sage.

Freeman, Miriam y Valentine, Deborah. (2004). Through the Eyes of Hollywood: Images of Social Workers in Film. Journal Social Work, 49(2), 151-161.

Gaughan, Lorraine y Garrett, Paul. (2012). The most twisted and unaccountable force in the state? Newspaper accounts of social work in the Republic of Ireland in troubled times. Journal of Social Work, 12, 267-286.

Gergen, Kenneth. (1999). An Invitation to Social Construction. Londres: Sage.

Gregory, Marilyn y Holloway, Margaret. (2005). The Debate as a Pedagogic Tool in Social Policy for Social Work Students. Social Work Education, 24(6), 617-637.

Guardiola, Dagmar y Serra Taylor, José. (2002). Política social y TS en Puerto Rico. Desafío y alternativas para el siglo XXI. San Juan: Publicaciones Puertorriqueñas.

Jiménez, Jillian; Mayers, Eileen; Chambers, Ruth y Pearlman, Cheryl. (2015). Social Policy and Social Change. Los Angeles: Sage.

Lens, Vicki. (2005). Advocacy and Argumentation in the Public Arena: A Guide for Social Workers. Social Work, 50(3), 231-238.

McCombs, Max. (1997). Building Consensus: The News Media's Agenda Setting Roles. Political Communication, 14, 433-443.

Mendes, Philip. (2001). Blaming the Messenger: The Media, Social Workers and Child Abuse. The Journal of the Australian Association of Social Workers, 54(2), 27-36.

Mink, Tarin y Twill, Sarah. (2012). Using Service-Learning to Teach a Social Work Policy Course. Journal of Community Engagement and Scholarship, 5(1). Recuperado de http://jces.ua.edu/ using-service-learning-to-teach-a-social-work-policy-course/ 
Montoya Lodoño, Catalina. (2009). Medios de comunicación y organizaciones de la sociedad civil: fortaleciendo alianzas para la democracia y los derechos humanos en América Latina. Signos y pensamientos, 25, 164-188.

NASW. (2008). Código de Ética. Recuperado de http://www.socialworkers.org/pubs/code/code.asp

Potter Jonathan. (2009). Discourse Analysis. En Melissa Hardy y Alan Bryman (Eds.), Handbook of Data Analysis (pp. 607-624). Londres: Sage.

Potter, Jonathan y Wetherell, Margaret. (1987). Discourse and Social Psychology: Beyond Attitudes and Behavior. Londres: Sage.

Rogers, Everett y Daring, James. (1994). Agenda setting research: where has it been, where is it going? En D. Graber (Ed.), Media Power in Politics (pp.77-95). Washington, DC: CQ Press.

Rozas Pagaza, Margarita. (s.f.). La intervención profesional, PS y derechos sociales claves para la construcción de la ciudadanía. Recuperado de http://www2.fices.unsl.edu.ar/ prosoc/documentos/ La_intervencion.doc

Rubio Ferreres, José María. (2009). Opinión pública y medios de comunicación. Teoría de la agenda setting. Gazeta de Antropología, 25(1).

Stone, Susan; Ekman, Eve; English, Dierdre y Fujimori, Sachi. (2008). Collaboration among Social Work and Journalism Students and Faculty. Journal of Social Work Education, 44(1), 163-172.

Vallespín, Fernando. (2000). La crisis de espacio público. Revista Española de Ciencias Políticas, 3,77-95.

Velasco Vázquez, Miren. (2012). Siete puertas para abrirnos a otro TS. Cuadernos de TS, 25(2), 471-476.

Von Sprecher, Roberto y Boito, María Eugenia. (2010). Comunicación y Trabajo Social. Córdova, Argentina: Editorial Brujas.

Weiss-Gal, Idit y Peled, Einat. (2007). Publishing Voice: Training Social Workers in Policy Practice. British Journal of Social Work, 39(2), 368-382.

Weiss-Gal, Idit y Gal, John. (2009). Realizing Rights in Social Work. Social Service Review, 83(2), 267-291.

Weiss-Gal, Idit y Gal, John. (2014). Social Workers as Policy Actors. Journal of Social Policy, 43 (1), 19-36.

Zurazaga, Carole; Surette, Raymond; Méndez, Mónica y Otto, Charles. (2006). Social Worker Perceptions of the Portrayal of the Profession in the Media. Journal of Social Work Education, 42(3), 621-636. 